\title{
Analisi dei parametri ipocentrali del terremoto del Friuli del 6 maggio 1976
}

\author{
Rodolfo Console e Calvino Gasparini \\ Annali di Geofisica, Vol. 29, n. 3, 1976.
}

\section{ABSTRACT}

The hypocentral parameters to $t_{0}, \phi_{0}, \lambda_{0}, h_{0}$, for the main shock occurred in Friuli on May 6th 1976 and itsfore shock were determined by the first arrival method. The computation was tried employing travel-time curves for different crustal models. The results show a very low influence of the travel-time curves on epicentral coordinates, whose error is essentially dependent on the precision of station data. The focal depth, on the contrary, is a parameter directly connected to the model used in the computation, so, if the model is not accurately known, the indetermination can be of the same order of magnitude as the crust thickness, even using very precise recorded data.

\section{Premessa}

Molto si è discusso sulla determinazione ipocentrale del terremoto del Friuli del 6 Maggio 1976. Limitandoci alle soluzioni ricavabili con il metodo degli inizi, in questo lavoro abbiamo illustrato i risultati che si ottengono utilizzando dromocrone di diverso modello.

\section{Il metodo}

Il metodo adoperato è quello degli inizi (onde $P g, P n, P$ ) per il quale abbiamo escluso le stazioni con distanza superiore a $1600 \mathrm{~km}$, ritenendo che i modelli regionali non sarebbero stati approssimati in modo utile oltre tale limite.

II metodo degli inizi si basa concettualmente sul principio della migliore ottimizzazione della relazione

$$
t_{s}=t_{0}+t\left(\phi_{0}, \lambda_{0}, h_{0}, \phi_{s}, \lambda_{s}\right)
$$

in cui $t_{s}$ è il tempo di arrivo delle prime onde, $t_{0}$ è il tempo origine del terremoto, $t$ è il tempo di tragitto dell'onda considerata, dipendente funzionalmente dalle coordinate $\phi_{0}$, $\lambda_{0}, h_{0}$ dell'ipocentro e da quelle $\phi_{s}, \lambda_{s}$ della stazione.

Partendo da una scelta provvisoria di $t_{0}, \phi_{0}, \lambda_{0}, h_{0} \mathrm{e}$ ottimizzando gli scarti col metodo dei minimi quadrati, si ottiene la soluzione quando per successive iterazioni non si hanno più variazioni significative dei parametri.

\section{I modelli}

Abbiamo utilizzato sia modelli sperimentali che sintetici.
I modelli sperimentali utilizzati (Figura 1) portano i nomi di «Di Filippo-Marcelli» la cui dromocrona venne ricavata dai dati provenienti dal terremoto del Gran Sasso del 1950 [Di Filippo e Marcelli 1952], "Caloi», ottenuto dal terremoto del Cansiglio [Caloi 1939], ed «HYPO 71» utilizzato ordinariamente dal Centre Séismologique Europeo-Méditerranéen di Strasburgo.

I modelli sintetici (Figura 2) portano convenzionalmente il nome di «Alpi» e «Appennini». Le relative dromocrone sono state ottenute ipotizzando teoricamente un modello medio crostale, aiutati anche dalle prospezioni sismiche attive, e dai dati sismici delle stazioni europee.

\section{Analisi dei risultati}

Sono stati calcolati gli epicentri per le scosse del 6 maggio 1976 delle ore 19.59, $M_{L}=4.5 \mathrm{RMP}$ (premonitoria) e delle ore 20.00, $M_{L}=6.2 \mathrm{RMP}$ (principale).

Per la scossa premonitoria abbiamo utilizzato i dati di 59 stazioni che avevano chiaramente registrato il primo arrivo, scartando quelle per cui ciò era discutibile.

Per la scossa principale sono state utilizzate solo 48 stazioni. Può sembrare strano che la scossa piu forte abbia dato luogo a un numero minore di registrazioni, ma non è così. L'intervallo di $1^{\mathrm{m}} 07^{\mathrm{s}}$ tra le due scosse ha fatto sì che l'inizio della scossa principale si trovasse per molte stazioni nel corpo della registrazione della scossa premonitoria (Figura 3) rendendone difficoltosa e imprecisa la lettura, specia1mente nel caso di deboli $P$ n.

Nelle Tabelle 1 e 2 sono riportati i dati del calcolo ipocentrale per i vari modelli. Il significato dei simboli usati in successione di scrittura è il seguente: sigla e nome delle stazioni; $F, L$, coordinate delle stazioni in gradi; $T$, tempo di registrazione in secondi contati dalle $19^{\mathrm{h}} 59^{\mathrm{m}} 00^{\mathrm{s}}$ per la scossa premonitoria e dalle $20^{\mathrm{h}} 00^{\mathrm{m}} 00^{\mathrm{s}}$ per la scossa principale; $V$, scarto sul tempo; $D$, distanza epicentrale in $\mathrm{km}$. I risultati dei calcoli sono riportati per righe in fondo alle tabelle 1 e 2 . Il significato dei simboli usati è il seguente: $T O$, tempo origine in secondi da sommare alle $19^{\mathrm{h}} 59^{\mathrm{m}} 00^{\mathrm{s}}$ per la scossa premonitoria e $20^{\mathrm{h}} 00^{\mathrm{m}} 00^{\mathrm{s}}$ per la scossa principale e relativo errore standard; FO, LO latitudine Nord e longitudine Est, dell'epicentro in gradi e relativo errore standard; $\mathrm{HO}$, profondità ipocentrale in km e relativo errore standard. 


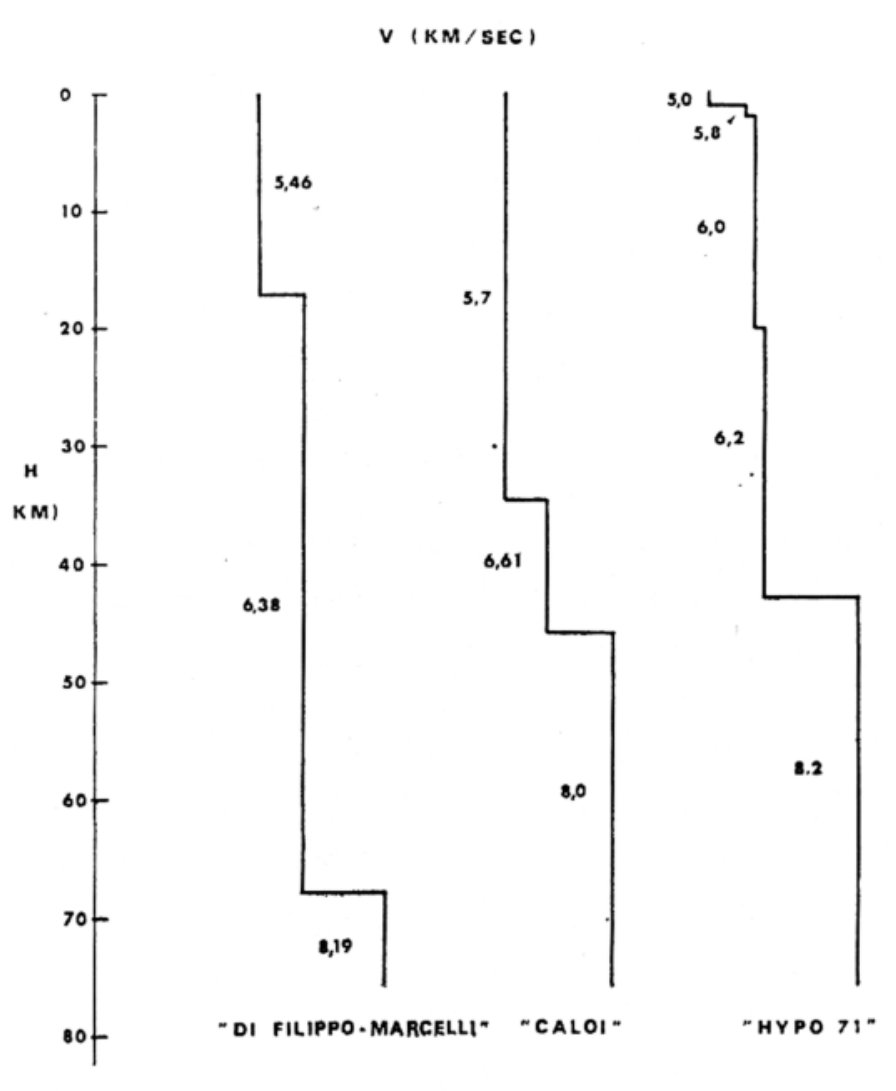

Figura 1. Modelli crostali delle dromocrone sperimentali.

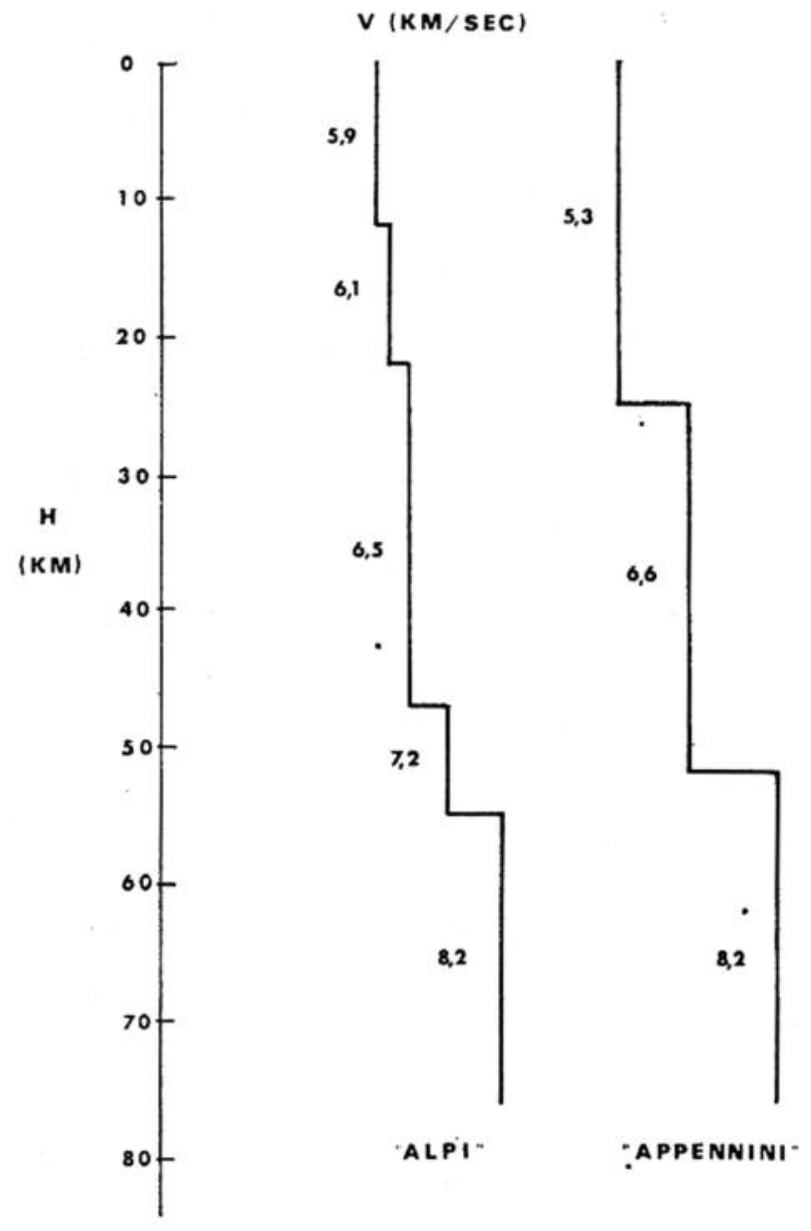

Dai risultati ottenuti si possono trarre diverse conclusioni significative:

a) il tempo origine viene influenzato dal modello in ragione maggiore dell'errore standard calcolato dal residuo dei tempi per le stazioni. Tuttavia la variazione è limitata a circa 1.5 secondi tra i casi estremi,

b) le coordinate epicentrali variano di poco rispetto al modello e il loro scarto massimo è contenuto nell'errore standard che è dell'ordine di 2/100 di grado,

c) la profondità ipocentrale è il parametro che varia più sensibilmente in relazione al modello dando valori estremamente diversi che scartano anche di decine di $\mathrm{km}$ mentre la relativa deviazione standard è contenuta entro qualche $\mathrm{km}$.

\section{Conclusione}

Dalle prove eseguite si può affermare che i dati ipocentrali calcolati sono affetti da errori provenienti da cause differenti.

Le coordinate epicentrali, con un modello più o meno realistico, conservano la loro posizione con uno scarto limitato. Quindi il loro errore dipende essenzialmente dall'accuratezza con cui i tempi vengono forniti dalle stazioni oppure da anomalie locali caratteristiche della stazione.

Con i metodi considerati, a meno di non conoscere a priori il modello da utilizzare, nulla si può dire sulla profondità ipocentrale tranne l'ordine di grandezza (crostale). Infatti le informazioni che derivano dagli scarti non permettono di individuare il modello più adatto al caso.

Per i due terremoti considerati è apparso chiaramente che la scossa premonitoria è avvenuta a profondità maggiore della principale, come risulta sistematicamente dal confronto dei risultati relativi allo stesso modello.

Disponendo di dati oggettivi ricavati indipendentemente per i parametri ipocentrali relativi a qualche terremoto, sarebbe possibile effettuare l'inversione dei dati stessi e quindi individuare il modello adatto per la zona considerata.

Tale ricerca sarà effettuata in un'ulteriore analisi riguardante i terremoti del Friuli con l'ausilio delle stazioni locali.

\section{Bibliografia}

Caloi, P. (1939). Tempi di tragitto per terremoti ad origine vicina, La Ricerca Scientifica, X, 5.

Di Filippo, D. e L. Marcelli (1952). Dromocrone per terremoti vicini e velocità delle onde nell'Italia Centrale, Annali di Geofisica, 5, n. 2.

(C) 2010 by the Istituto Nazionale di Geofisica e Vulcanologia. All rights reserved.

Figura 2. Modelli crostali delle dromocrone sintetiche. 


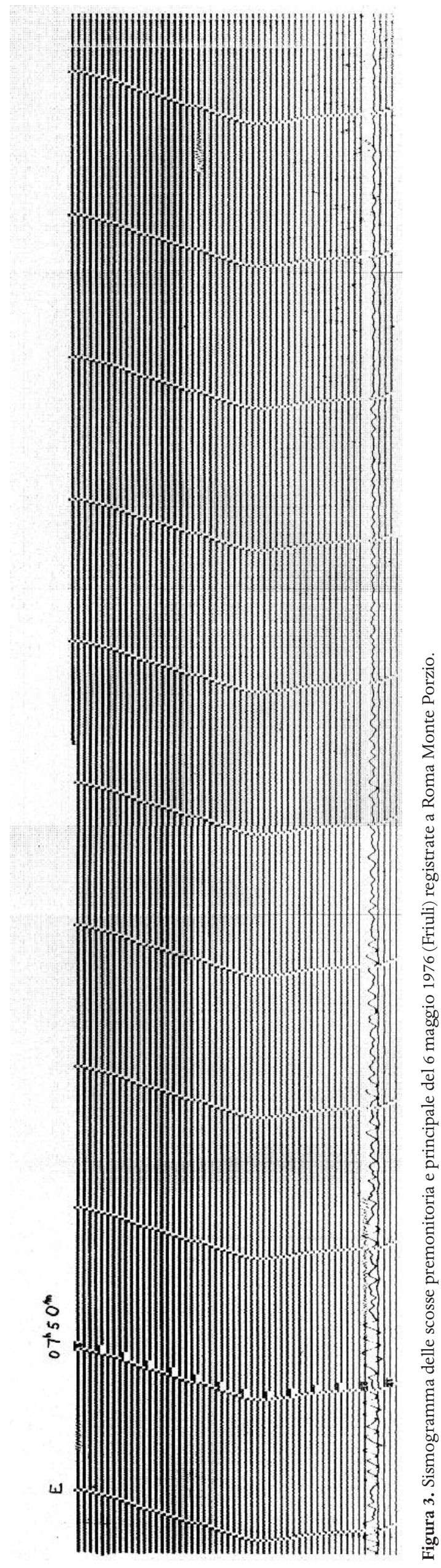




\begin{tabular}{|c|c|c|c|c|c|c|c|c|c|c|c|c|c|c|}
\hline \multicolumn{4}{|c|}{ Dati delle stazioni } & \multicolumn{3}{|c|}{ Mod. «Di Filippo-Marcelli» } & \multicolumn{2}{|c|}{ Mod. «Caloi» } & \multicolumn{2}{|c|}{ Mod. «HYPO 71» } & \multicolumn{2}{|c|}{ Mod. «Appennini» } & \multicolumn{2}{|c|}{ Mod. "Alpi» } \\
\hline & & $F$ & $L$ & $T$ & V & $D$ & V & $D$ & V & $D$ & V & $D$ & V & $D$ \\
\hline 1 & Ambiesta & 46.3797 & 12.9819 & 10.5 & 2.155 & 26.170 & .697 & 26.365 & .588 & 26.468 & .351 & 26.853 & 1.115 & 26.445 \\
\hline 2 & La Maina & 46.4483 & 12.7331 & 15.6 & -.714 & 46.744 & -1.410 & 46.892 & -1.286 & 47.008 & -1.291 & 47.392 & -1.082 & 47.014 \\
\hline 3 TRI & Trieste & 45.7089 & 13.7642 & 17.9 & .566 & 73.417 & .428 & 72.473 & .596 & 72.635 & .859 & 72.290 & .642 & 72.876 \\
\hline 4 & Mis & 46.1594 & 12.0811 & 21.0 & .483 & 94.261 & .924 & 93.621 & 1.022 & 93.965 & 1.477 & 94.261 & .875 & 94.200 \\
\hline 5 LJU & Ljunljana & 46.0433 & 14.5333 & 22.3 & -.022 & 99.564 & .652 & 99.670 & .658 & 99.473 & .970 & 99.105 & .353 & 99.388 \\
\hline 6 PAD & Padova & 45.4086 & 11.8861 & 32.0 & -2.629 & 145.918 & -1.522 & 144.665 & -1.479 & 145.134 & -1.713 & 145.244 & -2.225 & 145.531 \\
\hline 7 & Bolzano & 46.5049 & 11.3468 & 32.0 & -1.751 & 151.519 & -.615 & 151.311 & -.398 & 151.537 & -.698 & 151.897 & -1.307 & 151.652 \\
\hline 8 ZAG & Zagabria & 45.8167 & 15.9833 & 38.8 & 1.155 & 214.683 & .565 & 214.834 & 1.533 & 214.623 & 1.464 & 214.258 & 1.437 & 214.526 \\
\hline $9 \mathrm{SAL}$ & Salò & 45.6075 & 10.5262 & 41.3 & .476 & 226.983 & -.521 & 226.112 & .495 & 226.510 & .463 & 226.756 & .516 & 226.811 \\
\hline $10 \mathrm{BOL}$ & Bologna & 44.4867 & 11.3290 & 45.0 & -.178 & 251.658 & -1.180 & 250.290 & -.271 & 250.767 & -.251 & 250.803 & -.159 & 251.190 \\
\hline 11 FUR & Fürstenfeldbruck & 48.1655 & 11.2763 & 45.8 & -.077 & 259.118 & -.818 & 259.975 & .059 & 259.842 & .077 & 260.205 & 0.54 & 259.632 \\
\hline $12 \mathrm{CRN}$ & Corinaldo & 43.6308 & 12.9978 & 51.0 & -.836 & 295.355 & -1.742 & 293.947 & -.946 & 294.340 & -.987 & 294.155 & -.885 & 294.744 \\
\hline $13 \mathrm{PCN}$ & Piacenza & 45.0500 & 9.6667 & 52.5 & -.093 & 313.695 & -.891 & 312.704 & -.147 & 313.127 & -.147 & 313.337 & -.086 & 313.460 \\
\hline $14 \mathrm{KHC}$ & Kasperske Hory & 49.1306 & 13.5792 & 52.7 & .205 & 317.554 & -.340 & 318.959 & .306 & 318.568 & .304 & 318.758 & .278 & 318.166 \\
\hline $15 \mathrm{PRT}$ & Prato & 43.8800 & 11.0942 & 53.2 & -.254 & 317.866 & -1.139 & 316.465 & -.389 & 316.939 & -.414 & 316.945 & -.318 & 317.368 \\
\hline $16 \mathrm{RAV}$ & Ravensburg & 47.7833 & 9.6139 & 54.6 & -.721 & 325.478 & -1.359 & 325.821 & -.685 & 325.873 & -.696 & 326.263 & -.703 & 325.823 \\
\hline $17 \mathrm{PRG}$ & Perugia & 43.1014 & 12.3969 & 59.0 & -.935 & 360.274 & -1.655 & 358.833 & -1.014 & 359.257 & -1.054 & 359.114 & -.940 & 359.676 \\
\hline 18 MSS & Messtetten & 48.1792 & 8.9661 & 61.9 & -.243 & 389.460 & -.634 & 389.855 & -.180 & 389.890 & -.177 & 390.279 & -.223 & 389.825 \\
\hline 19 ZUR & Zurigo & 47.3686 & 8.5806 & 62.5 & -2.091 & 379.065 & -2.622 & 379.108 & -2.060 & 379.257 & -2.091 & 379.638 & -2.086 & 379.298 \\
\hline 20 GEN & Genova & 44.4181 & 8.9301 & 63.5 & -.654 & 399.369 & -1.221 & 398.285 & -.720 & 398.727 & -.686 & 398.907 & -.621 & 399.083 \\
\hline $21 \mathrm{STU}$ & Stuttgard & 48.7708 & 9.1933 & 64.8 & -.052 & 414.386 & -.416 & 414.995 & -.050 & 414.955 & -.007 & 415.338 & -.023 & 414.823 \\
\hline 22 PRU & Pruhonice & 49.9883 & 14.5417 & 65.1 & .633 & 422.596 & .410 & 424.040 & .732 & 423.613 & .703 & 423.752 & .682 & 423.192 \\
\hline 23 FEL & Feldberg & 47.8700 & 8.0167 & 67.6 & -.066 & 437.603 & -.381 & 437.783 & -.020 & 437.888 & -.026 & 438.275 & -.055 & 437.888 \\
\hline 24 & Tuscania & 42.4186 & 11.8744 & 68.0 & .263 & 443.652 & -.229 & 442.203 & .150 & 442.640 & .106 & 442.520 & .197 & 443.065 \\
\hline $25 \mathrm{BUH}$ & Buhlerhohe & 48.6756 & 8.2283 & 70.8 & .129 & 465.066 & -.098 & 465.518 & .122 & 465.533 & .163 & 465.923 & .152 & 465.450 \\
\hline $26 \mathrm{PNL}$ & Pinerolo & 44.9192 & 7.3139 & 72.0 & 1.923 & 490.007 & 1.679 & 489.186 & 1.882 & 489.573 & 1.866 & 489.830 & 1.882 & 489.860 \\
\hline $27 \mathrm{ROB}$ & Roburent & 44.2947 & 7.8706 & 72.5 & .083 & 478.844 & -.275 & 477.832 & .015 & 478.271 & -.032 & 478.465 & 0.30 & 478.598 \\
\hline $28 \mathrm{HEI}$ & Heidelberg & 49.3986 & 8.7264 & 73.4 & .103 & 486.512 & .021 & 498.204 & .189 & 487.134 & .180 & 487.513 & .136 & 486.976 \\
\hline 29 RMP & Romamonteporzio & 41.8111 & 12.7023 & 74.0 & .979 & 498.756 & .666 & 497.342 & .862 & 497.740 & .844 & 497.560 & .957 & 498.146 \\
\hline $30 \mathrm{STR}$ & Strasbourg & 48.5847 & 7.7658 & 74.3 & -.502 & 488.969 & -.600 & 489.344 & -.441 & 489.386 & -.440 & 489.777 & -.482 & 489.327 \\
\hline 31 SAR & Sarajevo & 43.8733 & 18.4283 & 74.5 & -1.202 & 484.802 . & -1.433 & 484.478 & -1.237 & 484.418 & -1.350 & 484.029 & -1.266 & 484.462 \\
\hline 32 MOX & Moxa & 50.6461 & 11.6461 & 75.0 & .176 & 500.362 & 1.92 & 501.593 & .289 & 501.300 & .342 & 501.583 & .314 & 500.965 \\
\hline 33 STV & S. Ann & 44.2442 & 7.3244 & 75.2 & 2.398 & 519.806 & 2.144 & 518.829 & 2.285 & 519.250 & 2.288 & 519.465 & 2.348 & 519.579 \\
\hline 34 WLS & Welschbruch & 48.4128 & 7.3536 & 75.8 & .250 & 507.127 & .149 & 507.413 & .231 & 507.485 & .297 & 507.874 & .293 & 507.452 \\
\hline $35 \mathrm{BSF}$ & Ballon Servance & 47.8333 & 6.7939 & 76.0 & 1.938 & 522.634 & 1.837 & 522.710 & 1.936 & 522.850 & 1.940 & 523.232 & 1.944 & 522.880 \\
\hline $36 \mathrm{CDF}$ & Champ du Feu & 48.3941 & 7.2707 & 76.7 & -.064 & 511.784 & -168 & 512.058 & -.101 & 512.133 & -.045 & 512.523 & -.049 & 512.105 \\
\hline $37 \mathrm{ECH}$ & Echery & 48.2158 & 7.1583 & 77.5 & -.911 & 511.397 & -1.022 & 511.611 & -.954 & 511.707 & -.896 & 512.094 & -.898 & 511.696 \\
\hline $38 \mathrm{HAU}$ & Haudompre & 48.0054 & 6.3500 & 82.5 & .019 & 559.979 & -.005 & 560.078 & -.041 & 560.210 & .024 & 560.593 & .027 & 560.235 \\
\hline 39 CLL & Collmberg & 51.3090 & 13.0044 & 82.6 & -.137 & 559.544 & .002 & 560.902 & -.099 & 560.542 & -.056 & 560.766 & -.082 & 560.159 \\
\hline $40 \mathrm{BEO}$ & Beograd & 44.8214 & 20.4553 & 86.0 & -.813 & 582.210 & -.711 & 582.340 & -.854 & 582.137 & -.935 & 581.770 & -.856 & 582.045 \\
\hline $41 \mathrm{SPF}$ & St. Paul en F. & 43.5639 & 6.6961 & 86.8 & .795 & 601.448 & .736 & 600.396 & .633 & 600.832 & .617 & 601.023 & .740 & 601.181 \\
\hline 42 LMR & La Mourre & 43.3333 & 6.5092 & 89.9 & .894 & 628.102 & .999 & 627.030 & .774 & 627.469 & .771 & 627.653 & .837 & 627.823 \\
\hline 43 LRG & Lorgues & 43.4542 & 6.3606 & 90.6 & .529 & 630.888 & .664 & 629.842 & .413 & 630.278 & .409 & 630.471 & .473 & 630.624 \\
\hline 44 TTG & Titograd & 42.4297 & 19.2608 & 92.5 & -.318 & 639.610 & -.125 & 639.086 & -.380 & 639.095 & -.503 & 638.708 & -.398 & 639.200 \\
\hline $45 \mathrm{STB}$ & Steinbach & 50.5953 & 6.8400 & 96.8 & -.130 & 676.235 & .317 & 676.936 & -.098 & 676.863 & -.079 & 677.241 & -.097 & 676.702 \\
\hline 46 BNS & Bensberg & 50.9639 & 7.1756 & 98.2 & -.076 & 688.300 & .466 & 689.085 & .005 & 688.980 & -.026 & 689.351 & -.046 & 688.793 \\
\hline $47 \mathrm{JUE}$ & Julich & 50.9139 & 6.4098 & 104.6 & -2.268 & 722.591 & -1.686 & 723.302 & -2.235 & 723.225 & -2.215 & 723.602 & -2.234 & 723.061 \\
\hline 48 TIR & Tirana & 41.3467 & 19.8667 & 106.8 & .265 & 761.198 & .761 & 760.585 & .111 & 760.626 & .082 & 760.243 & .189 & 760.758 \\
\hline $49 \mathrm{LBF}$ & Les Buteaux & 46.9869 & 3.9772 & 101.3 & .355 & 716.948 & .791 & 716.722 & .299 & 716.953 & .330 & 717.310 & .347 & 717.072 \\
\hline 50 SSF & Saint Saulge & 47.0614 & 3.5068 & 105.8 & .292 & 753.086 & .829 & 752.872 & .205 & 753.099 & .225 & 753.458 & .216 & 753.215 \\
\hline $51 \mathrm{SKO}$ & Skopje & 41.9721 & 21.4396 & 113.5 & -.693 & 808.210 & .004 & 807.843 & -.831 & 807.798 & -.875 & 807.408 & -.780 & 807.855 \\
\hline $52 \mathrm{OHR}$ & Ohrid & 41.1114 & 20.7989 & 115.3 & .583 & 833.508 & 1.340 & 832.962 & .452 & 832.979 & .363 & 832.529 & .469 & 833.091 \\
\hline $53 \mathrm{TCF}$ & Toulx Ste Croix & 46.2881 & 2.2139 & 117.5 & .760 & 852.821 & 1.579 & 852.455 & .660 & 852.725 & .675 & 853.067 & .674 & 852.886 \\
\hline $54 \mathrm{VAY}$ & Valandovo & 41.3211 & 22.5701 & 127.5 & -.253 & 926.837 & .827 & 926.476 & -.367 & 926.429 & -.423 & 926.039 & -.324 & 926.483 \\
\hline $55 \mathrm{LPO}$ & Le Pouchou & 44.6833 & 1.1872 & 131.9 & -.385 & 961.615 & .715 & 961.003 & -.506 & 961.340 & -.505 & 961.642 & -.413 & 961.569 \\
\hline $56 \mathrm{LFF}$ & La Frestale & 44.9375 & 360.7364 & 135.6 & -.658 & 989.383 & .531 & 988.820 & -.825 & 989.143 & -.813 & 989.454 & -.779 & 989.359 \\
\hline $57 \mathrm{MFF}$ & St. Martin D.F. & 46.6014 & -360.1433 & 139.6 & .511 & 1031.578 & 1.838 & 1031.280 & .362 & 1031.531 & .378 & 1031.882 & .399 & 1031.672 \\
\hline $58 \mathrm{FLN}$ & Foliniere & 48.7625 & -360.4819 & 145.1 & -.167 & 1071.578 & 1.378 & 1071.610 & -.247 & 1071.763 & -.190 & 1072.143 & -.185 & 1071.807 \\
\hline
\end{tabular}

Tabella 1. Terremoto del Friuli, 6 maggio 1976, ore 19.59 (premonitoria).

Valori Modello «HYPO 71»

$\mathrm{TO}=6.103+/-.285 \quad F O=46.272+/-.015 \quad L O=13.288+/-.019$

$\mathrm{HO}=12.248+/-2.504$

Valori Modello «Appennini»

Valori Modello «Di Filippo-Marcelli»

$\mathrm{TO}=4.498+/-.235 \quad F O=46.281+/-.016 \quad L O=13.290+/-.021$

$\mathrm{TO}=4.708+/-.359 \quad F O$

$H O=18.256+/-2.466$

Valori Modello «Alpi»

$\mathrm{TO}=5.881+/-.312 \quad F O=46.275+/-.015 \quad L O=13.291+/-.020$

Valori Modello "Caloi»

$T O=4.952+/-.317 \quad F O=46.269+/-.017 \quad L O=13.284+/-.0222$

$H O=21.829+/-3.120$

$H O=23.894+/-2.629$ 


\begin{tabular}{|c|c|c|c|c|c|c|c|c|c|c|c|c|c|c|}
\hline \multicolumn{4}{|c|}{ Dati delle stazioni } & \multicolumn{3}{|c|}{ Mod. «Di Filippo-Marcelli» } & \multicolumn{2}{|c|}{ Mod. «Caloi» } & \multicolumn{2}{|c|}{ Mod. «HYPO 71» } & \multicolumn{2}{|c|}{ Mod. «Appennini» } & \multicolumn{2}{|c|}{ Mod. «Alpi» } \\
\hline & & $F$ & $L$ & $T$ & $V$ & $D$ & V & $D$ & V & $D$ & V & $D$ & V & $D$ \\
\hline 1 & La Maina & 46.4483 & 12.7339 & 19.5 & 1.565 & 43.644 & .808 & 44.649 & .929 & 44.180 & .348 & 44.582 & .928 & 44.132 \\
\hline $2 \mathrm{VAJ}$ & Vajont & 46.2661 & 12.3302 & 22.7 & 1.977 & 69.112 & 2.018 & 70.932 & 1.990 & 69.652 & 1.867 & 70.131 & 1.970 & 69.439 \\
\hline 3 TRI & Trieste & 45.7089 & 13.7642 & 24.8 & .537 & 73.532 & .366 & 73.565 & .453 & 73.104 & .284 & 72.855 & .474 & 72.996 \\
\hline 4 & Pieve di Cadore & 46.4208 & 12.3869 & 25.6 & -1.196 & 67.243 & -1.266 & 68.678 & -1.220 & 67.802 & -1.373 & 68.255 & -1.231 & 67.673 \\
\hline 5 & Mis & 46.1594 & 12.0819 & 30.1 & -2.481 & 88.950 & -1.982 & 90.931 & -2.117 & 89.471 & -1.768 & 89.949 & -2.161 & 89.218 \\
\hline $6 \mathrm{LJU}$ & Ljunljana & 46.0433 & 14.5333 & 30.2 & -.316 & 103.627 & -.179 & 102.121 & .047 & 103.072 & .520 & 102.604 & .079 & 103.227 \\
\hline 7 ZAG & Zagabria & 45.8167 & 15.9833 & 48.4 & -.611 & 218.844 & -1.477 & 217.309 & .344 & 218.287 & .297 & 217.823 & -.089 & 218.443 \\
\hline $8 \mathrm{CRN}$ & Corinaldo & 43.6308 & 12.9978 & 57.0 & .735 & 292.018 & -.544 & 293.419 & .779 & 291.910 & .766 & 291.967 & .756 & 291.610 \\
\hline 9 PRT & Prato & 43.8800 & 11.0942 & 60.7 & -.479 & 312.658 & -1.585 & 314.741 & -.410 & 312.834 & -.384 & 313.119 & -.439 & 212.473 \\
\hline 10 FIR & Firenze Xim & 43.7739 & 11.2550 & 62.0 & -1.326 & 316.332 & -2.450 & 318.367 & -1.273 & 316.479 & -1.236 & 316.741 & -1.302 & 316.120 \\
\hline $11 \mathrm{VIE}$ & Vienna & 48.2483 & 16.3617 & 62.0 & -.277 & 324.710 & -1.930 & 322.495 & -.286 & 324.424 & -.320 & 324.062 & -.262 & 324.781 \\
\hline $12 \mathrm{PAV}$ & Pavia & 45.1833 & 9.1736 & 63.7 & -.451 & 337.136 & -1.539 & 339.299 & -.363 & 337.598 & -.302 & 338.060 & -.287 & 337.297 \\
\hline 13 TNO & Torino & 45.0587 & 7.6969 & 75.0 & 2.093 & 450.815 & 1.397 & 452.929 & 2.205 & 451.298 & 2.242 & 451.767 & 2.188 & 451.010 \\
\hline 14 & Tuscania & 42.4186 & 11.8744 & 75.5 & .232 & 439.492 & -.598 & 441.200 & .260 & 439.490 & .287 & 439.636 & .257 & 439.157 \\
\hline $15 \mathrm{BUH}$ & Buhlerhohe & 48.6755 & 8.2284 & 78.5 & .098 & 462.936 & -.783 & 463.627 & .164 & 463.451 & .202 & 463.816 & .181 & 463.449 \\
\hline 16 RMP & Romamonteporzio & 41.8111 & 12.7023 & 81.0 & 1.531 & 495.318 & .857 & 496.760 & 1.565 & 495.223 & 1.565 & 495.291 & 1.525 & 494.919 \\
\hline $17 \mathrm{HEI}$ & Heidelberg & 49.3986 & 8.7264 & 81.5 & -.161 & 485.387 & -1.015 & 485.684 & -.103 & 485.856 & -.031 & 486.160 & -.104 & 485.918 \\
\hline $18 \mathrm{SAR}$ & Sarajevo & 43.8733 & 18.4283 & 82.1 & -.514 & 487.446 & -1.505 & 486.531 & -.569 & 486.911 & -.588 & 486.512 & -.580 & 486.952 \\
\hline $19 \mathrm{BEO}$ & Beograd & 44.8214 & 20.4553 & 94.5 & -.851 & 586.301 & 1.630 & 584.800 & -.918 & 585.743 & -.966 & 585.282 & -.907 & 585.893 \\
\hline 20 TTG & Titograd & 42.4297 & 19.2608 & 98.5 & 1.877 & 641.492 & 1.360 & 640.882 & 1.846 & 640.984 & 1.806 & 640.629 & 1.812 & 640.974 \\
\hline 21 STB & Steinbach & 50.5953 & 6.8400 & 104.2 & .310 & 675.144 & -.003 & 675.429 & .323 & 675.611 & .361 & 675.914 & .367 & 675.676 \\
\hline 22 BNS & Bensberg & 50.9639 & 7.1756 & 105.8 & .203 & 687.591 & -.050 & 687.726 & .275 & 688.036 & .317 & 688.313 & .261 & 688.125 \\
\hline $23 \mathrm{JUE}$ & Julich & 50.9139 & 6.4098 & 110.2 & -.022 & 721.542 & -.219 & 721.811 & -.009 & 722.007 & .016 & 722.306 & .035 & 722.074 \\
\hline 24 LOR & Lormes & 47.2683 & 3.8589 & 110.2 & .266 & 723.944 & .257 & 725.472 & .290 & 724.501 & .344 & 724.964 & .308 & 724.347 \\
\hline 25 BEG & Berghein & 50.9613 & 6.6557 & 110.5 & -1.375 & 712.770 & -1.596 & 712.990 & -1.363 & 713.228 & -1.347 & 713.520 & -1.318 & 713.303 \\
\hline $26 \mathrm{SSF}$ & Saint Saulge & 47.0614 & 3.5068 & 113.0 & .477 & 748.782 & .589 & 750.365 & .546 & 749.338 & .607 & 749.805 & .578 & 749.173 \\
\hline $27 \mathrm{LBF}$ & Les Buteaux & 46.9869 & 3.9772 & 108.6 & .506 & 712.611 & .451 & 714.208 & .530 & 713.167 & .567 & 713.635 & .546 & 712.999 \\
\hline 28 TIR & Tirana & 41.3467 & 19.8667 & 114.5 & .717 & 762.707 & .538 & 762.246 & .616 & 762.216 & .556 & 761.884 & .648 & 762.180 \\
\hline $29 \mathrm{DEV}$ & Deva & 45.8833 & 22.9028 & 115.4 & -1.871 & 749.203 & -2.198 & 747.354 & -1.935 & 748.665 & -1.988 & 748.184 & -1.859 & 748.887 \\
\hline 30 PAVF & & 46.7919 & 3.3569 & 115.4 & -.624 & 759.076 & -.561 & 760.721 & -.596 & 759.630 & -.558 & 760.101 & -.580 & 759.451 \\
\hline 31 SKO & Skopje & 41.9721 & 21.4396 & 120.5 & .575 & 810.686 & .525 & 809.841 & .468 & 810.156 & .403 & 809.768 & .508 & 810.185 \\
\hline $32 \mathrm{OHR}$ & Ohrid & 41.1114 & 20.7989 & 121.9 & 2.145 & 835.289 & 2.212 & 834.721 & 2.024 & 834.786 & 2.027 & 834.437 & 2.058 & 834.768 \\
\hline $33 \mathrm{TCF}$ & Toulx Ste Croix & 46.2881 & 2.2100 & 126.2 & -.506 & 848.432 & -.133 & 850.176 & -.505 & 848.980 & -.435 & 849.456 & -.473 & 848.781 \\
\hline 34 MES & Messina Uni & 38.1989 & 15.5550 & 132.5 & 1.258 & 914.710 & 1.712 & 915.550 & 1.184 & 914.450 & 1.154 & 914.368 & 1.192 & 914.220 \\
\hline $35 \mathrm{RCI}$ & Reggio Calabria & 38.1056 & 15.6433 & 134.4 & .760 & 926.399 & 1.285 & 927.226 & .686 & 926.136 & .656 & 926.050 & .695 & 925.907 \\
\hline $36 \mathrm{VAY}$ & Valandovo & 41.3211 & 22.5701 & 134.6 & .913 & 929.333 & 1.248 & 928.481 & .806 & 928.803 & .740 & 928.413 & .846 & 928.833 \\
\hline 37 GIB & Gibilmanna & 37.9833 & 14.0167 & 135.7 & -1.231 & 920.635 & -.724 & 921.775 & -1.296 & 920.451 & -1.317 & 920.440 & -.1292 & 920.182 \\
\hline 38 LPO & Le Pouchou & 44.6833 & 1.1872 & 140.3 & -1.439 & 956.403 & -.750 & 958.373 & -1.427 & 956.923 & -1.381 & 957.403 & -1.447 & 956.673 \\
\hline 39 LFF & La Frestale & 44.9375 & 360.7364 & 143.1 & -.853 & 984.264 & -.049 & 986.193 & -.875 & 984.792 & -.818 & 985.272 & -.868 & 984.552 \\
\hline $40 \mathrm{MFF}$ & St. Martin D.F. & 46.6014 & -360.1433 & 146.8 & .664 & 1027.057 & 1.581 & 1028.731 & .663 & 1027.609 & .700 & 1028.082 & .677 & 1027.425 \\
\hline 41 BUC & Bucharest & 44.4136 & 26.0967 & 150.0 & -2.421 & 1027.992 & -1.933 & 1026.317 & -2.557 & 1027.440 & -2.634 & 1026.967 & -2.499 & 1027.625 \\
\hline 42 FLN & Foliniere & 48.7625 & -360.4819 & 152.6 & -.150 & 1067.981 & .851 & 1069.273 & -.126 & 1068.537 & -.092 & 1068.979 & -.103 & 1068.428 \\
\hline $43 \mathrm{LPF}$ & Le Petre & 48.0317 & -1.0408 & 155.2 & .999 & 1098.386 & 2.024 & 1099.819 & .990 & 1098.943 & 1.054 & 1099.398 & .943 & 1098.807 \\
\hline $44 \mathrm{MBZ}$ & Menzel B. Zelfa & 36.6739 & 10.6722 & 156.3 & -1.794 & 1084.574 & -.746 & 1086.217 & -1.904 & 1084.548 & -1.892 & 1084.675 & -1.908 & 1084.221 \\
\hline $45 \mathrm{ZGN}$ & Zaghouan & 36.3750 & 10.1117 & 160.4 & -.742 & 1127.015 & .479 & 1128.716 & -.809 & 1127.011 & -.811 & 1127.155 & -.813 & 1126.678 \\
\hline 46 IST & Istanbul & 41.0456 & 28.9958 & 191.2 & 1.077 & 1394.040 & 2.671 & 1392.712 & .868 & 1393.484. & .806 & 1393.038 & .918 & 1393.600 \\
\hline 47 UPP & Uppsala & 59.8583 & 17.6267 & 208.5 & 1.833 & 1542.001 & 3.803 & 1540.439 & 1.696 & 1542.055 & 1.672 & 1541.953 & 1.771 & 1542.373 \\
\hline $48 \mathrm{CIN}$ & Cine & 37.6000 & 28.0867 & 214.0 & -1.653 & 1558.108 & .416 & 1557.255 & -1.884 & 1557.577 & $\mid-1.925$ & 1557.188 & $\mid-1.901$ & 1557.607 \\
\hline
\end{tabular}

Tabella 2. Terremoto del Friuli, 6 maggio 1976, ore 20.00 (principale).

Valori Modello «HYPO 71»

$\mathrm{TO}=12.797+/-.670 \quad F O=46.253+/-.023 \quad L O=13.234+/-.025$ $\mathrm{HO}=1.231+/-5.570$

Valori Modello «Appennini»

$\mathrm{TO}=11.278+/-.446 \quad F O=46.253+/-.023 \quad L O=13.240+/-.024$

$\mathrm{HO}=8.194+/-3.205$

Valori Modello «Alpi»

$\mathrm{TO}=12.585+/-.198 \quad \mathrm{FO}=46.250+/-.023 \quad L O=13.231+/-.025$

$\mathrm{HO}=9.995+/-.014$
$\mathrm{TO}=11.756+/-.449 \quad \mathrm{FO}=46.266+/-.027 \quad L O=13.250+/-.029$ $\mathrm{HO}=20.088+/-3.797$ 